\title{
IN-FLIGHT OXYGEN COLLECTION FOR A TWO-STAGE AIR-LAUNCH VEHICLE: INTEGRATION OF VEHICLE AND SEPARATION CYCLE DESIGN
}

\author{
D. Verstraete, D. Bizzarri, and P. Hendrick
}

In-flight oxygen collection is a very promising technique to reduce the launch costs and improve the payload capabilities of two-stage-to-orbit semireusable launchers. Using liquid hydrogen the incoming air is deeply cooled and enriched in oxygen during the cruise phase of the first stage. The liquified enriched air is stored in the second stage which is then launched into orbit. This paper gives the result of a conceptual design of a two-stage-to-orbit air launched space vehicle. The mass, aerodynamic, and propulsive characteristics of the first stage are determined and an assessment of the influence of the collection plant performance on the subsonic first stage is made. The results for a centrifugally enhanced destillation unit are given together with the plant cycle arrangement. Integration options for the plant into the first stage are proposed and a short description of the air separation test unit and its test bench is also given.

\section{INTRODUCTION}

In-flight oxygen collection is a conceptual design approach of orbital launchers that has the potential to significantly improve the payload capacity of space launch vehicles with technologies available in medium to short term. With inflight oxygen collection, the cooling capacity of the liquid hydrogen fuel is used during the first air-breathing flight phase. The hydrogen is used to deeply cool a fraction of the incoming air and enrich it in oxygen. The liquified enriched air (LEA) is then stored in the second stage - a reusable or expandable second stage orbiter, which is consequently launched. The main advantages of the concept, applied here to the 3rd generation two-stage-to-orbit launcher, are a much reduced takeoff mass, a reduced mass for the liquid rocket engines, and a much increased operational flexibility in terms of reachable orbit and launch window $[1]$.

This is an Open Access article distributed under the terms of the Creative Commons Attribution-Noncommercial License 3.0, which permits unrestricted use, distribution, and reproduction in any noncommercial medium, provided the original work is properly cited. 
This paper presents the integrated result of research on both the hydrogen fueled airplane and the $\mathrm{O}_{2}-\mathrm{N}_{2}$ separation device and cycle performance prediction. The proposed double fuselage carrier, which uses hydrogen as a fuel, would collect the LEA, needed afterwards to propel the all-rocket orbiter, during an extended cruise phase that precedes the subsonic air launch of the orbiter. The results from a conceptual design with the Advanced Aircraft Analysis (AAA) software from Darcorp (USA) of the mothership are given first. The influence of the collection-plant collection ratio is assessed too. On the propulsion/separation cycle integration side, the paper concentrates on the study of a centrifugal distillation device. From a theoretical viewpoint, the internal arrangement of the separator is adapted to be integrated in the subsonic twin fuselage carrier. Previous results are used to size the separation device and show that it can be integrated inside the twin fuselage first stage.

\section{CONCEPTUAL DESIGN OF THE TWIN FUSELAGE MOTHERSHIP}

The conceptual design of the mothership was performed using AAA software from Darcorp $[2,3]$. As the methodology from the software is followed completely, it is not repeated here. Only the necessary modifications to take the unconventional configuration into account are briefly commented upon as they have been published previously [4]. The whole design process is built around the mission specification for the aircraft under consideration $[5,6]$. The design mission for the first stage, called the mothership, is therefore given first and some particularities of the assumptions are then commented. Thereafter, a preliminary estimate of the aircraft takeoff gross weight (TOGW or $W_{\mathrm{TO}}$ ) and empty weight $\left(W_{E}\right)$ is made, as it is needed in the performance sizing procedure, which is described in subsection 2.3. Here, the drag polars of the mothership and orbiter are given too. Finally, the influence of the collection ratio (C.R.) on the design of the mothership is assessed. The collection ratio is the amount of LOX collected per kilogram of hydrogen fuel used.

\subsection{Mission Specification for the Mothership}

The mission specification used to design the mothership is summarized in Table 1 and graphically represented in Fig. 1. The design mission range is selected based on the following considerations. First of all, a suitable airport to takeoff and land is selected. Runway limitations could namely easily form an obstacle for an aircraft of the envisaged size. Therefore, Vandenberg Air Force Base, near Los Angeles (see Fig. 1) has been chosen as the operating base for the mothership. 
Table 1 The mission specification

\begin{tabular}{ll}
\hline \multicolumn{1}{c}{ Parameter } & \multicolumn{1}{c}{ Specification } \\
\hline $\begin{array}{l}\text { Role } \\
\text { Payload }\end{array}$ & 3rd generation reusable launcher \\
Orbiter & $W_{\text {TO, orb }}=90 \mathrm{t}$ \\
LOX & $W_{\mathrm{LOX}}=210 \mathrm{t}($ collected during cruise $)$ \\
LCP & $W_{\mathrm{LCP}}=10 \mathrm{t}$ \\
Crew & $2 \times 2$ in cockpit $/ 2 \times 1$ for the LCP \\
Engines & 6 GE90-90B engines running on liquid hydrogen $\left(\mathrm{LH}_{2}\right)$ \\
Performance & \\
Range & Still air range in cruise of $12,500 \mathrm{~km}$ \\
Speed & Mach 0.76 at $30,000 \mathrm{ft}$ \\
Climb & Direct climb to $30,000 \mathrm{fr}$ \\
\hline
\end{tabular}

After all, this airport has a runway length of $15,000 \mathrm{ft}(4,572 \mathrm{~m})$ and is located at only $367 \mathrm{ft}$ above sea level, which is favorable for the engine thrust at takeoff. A launching point has, of course, to be chosen too for the orbiter in order to fix the design range. The Boeing Sea Launch point is chosen as it is the only known launch point on the equator and it furthermore enables a wide range for the launch azimuth. Some extra range is added to the great circle distance between both points so that a spatial window around the equator is assured for the launch. This can be seen in Fig. 1 where the curve represents the points of no return. As the aircraft is assumed to take off from its base, fly to the launch point and return to the base, the design mission range is thus fixed.

For the propulsion, 6 GE90-90B engines running on hydrogen are selected. Hydrogen is chosen as a fuel for the engines as it is needed anyway to chill down the air for the separation of oxygen and its liquefaction. It has the additional benefit of reducing the fuel weight by a factor of approximately 3 . However, the weight of the insulation needed to store the cryogenic $\mathrm{LH}_{2}$ partly reduces this weight advantage. Nevertheless, for missions with a large range, the use of $\mathrm{LH}_{2}$ lowers the TOGW significantly. Due to its very low density $\left(70.8 \mathrm{~kg} / \mathrm{m}^{3}\right)$, however, a much bigger volume is needed to store the fuel (approximately 4 times the volume needed to store kerosene for a mission with the same range [7]). The range envisaged for the mothership mission thus obliges the use of a twin fuselage layout, most probably with the fuselages built around the insulated $\mathrm{LH}_{2}$ tanks.

The collection of the LOX, needed to propel the orbiter and its payload to orbit, takes place just before the launch of the orbiter. This is done to keep the weight of the airplane during the mission as low as possible which consequently reduces the fuel consumed during the complete mission. The weight of the orbiter at takeoff is set to $90 \mathrm{t}$ of which $35 \mathrm{t}$ are liquid hydrogen needed for the second stage propulsion into orbit. With these weights, a payload of $13.4 \mathrm{t}$ can be 


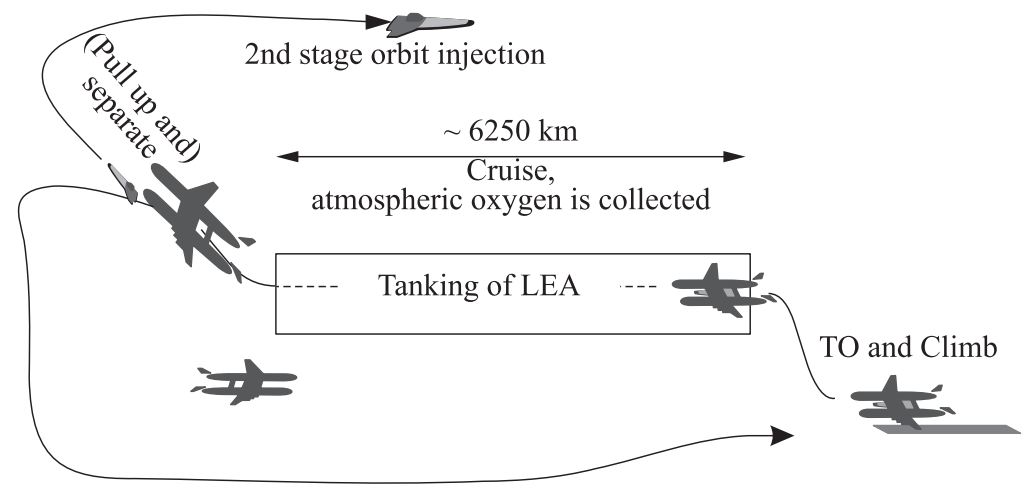

1st stage returns directly to Base

(a)

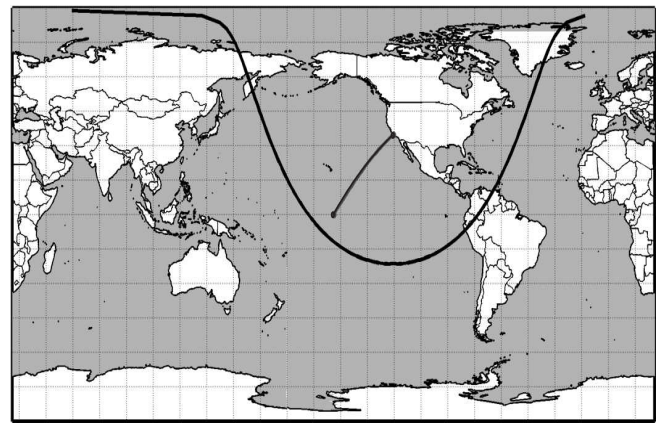

(b)

Figure 1 Mission profile used to size the mothership: (a) the mission profile and (b) typical mothership mission

brought into a lower Earth orbit at an inclination of $5^{\circ}$. When the orbit has an inclination of $98^{\circ}$, the payload is reduced to $9.2 \mathrm{t}$ [8].

\subsection{Estimation of the Takeoff Gross Weight, Empty Weight, and Mission Fuel Weight}

To determine the TOGW $\left(W_{\mathrm{TO}}\right)$ and the empty weight $\left(W_{E}\right)$, statistical relationships are typically used during the conceptual design phase [5]. In AAA, the following relationship is imposed:

$$
\log _{10} W_{\mathrm{TO}}=A+B \log _{10} W_{E}
$$




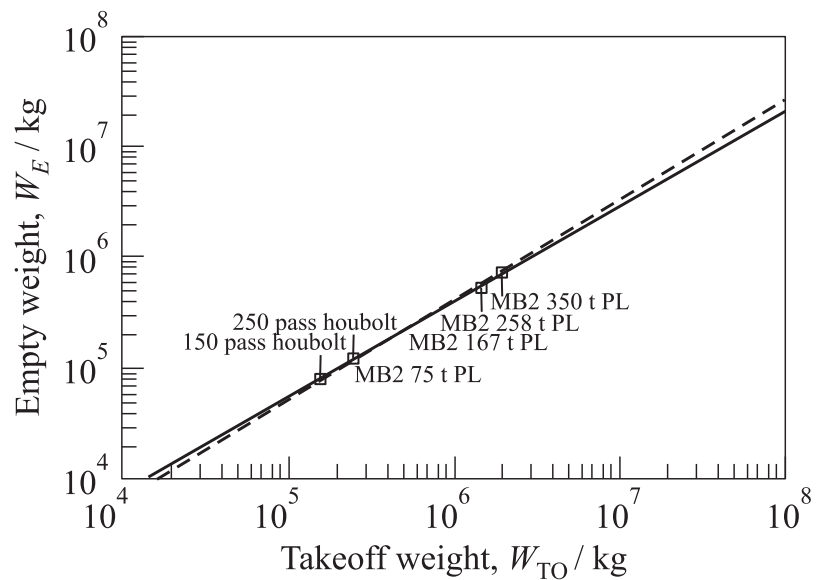

(a)

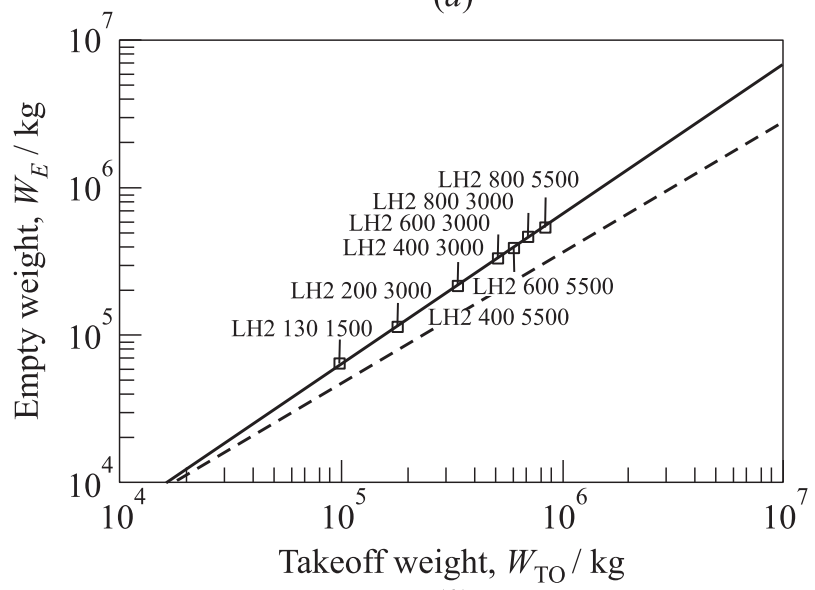

(b)

Figure 2 Regression coefficients $A$ and $B$ : $(a)$ twin fuselage aircraft and (b) hydrogenfuelled aircraft

where the so-called regression coefficients $A$ and $B$ are determined per aircraft category. As the envisaged configuration differs too much from the proposed aircraft categories, new regression coefficients are determined. Since two almost independent configuration changes are made, this problem is subdivided into two separate subproblems. First, the influence of the twin fuselage layout on the regression coefficients $A$ and $B$ is examined. New regression coefficients are determined through a least squares approximation based on available data on twin fuselage designs [9-11] as shown in Fig. $2 a$. As shown in the figure, the empty weight fraction of twin fuselage aircraft (solid line) lies close to the ones 


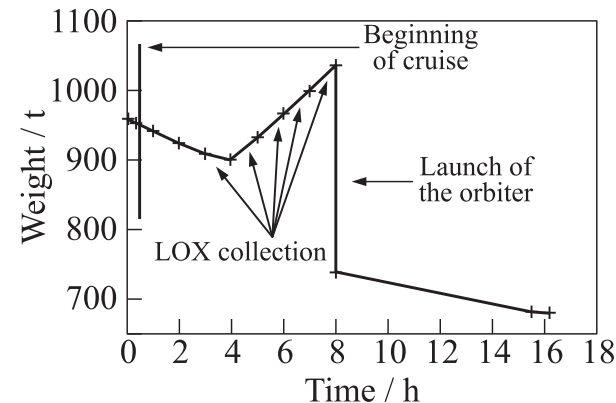

(a)

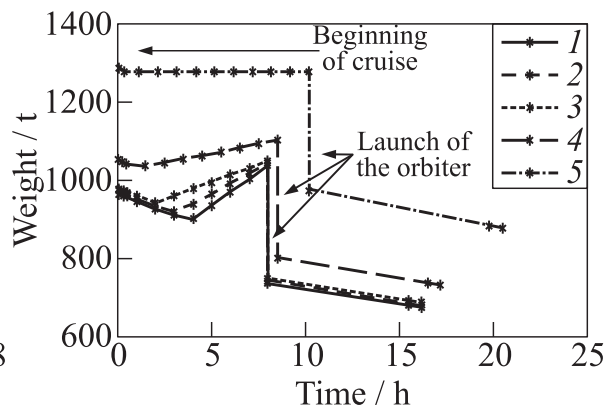

(b)

Figure 3 Evolution of the weight during the mission: $(a)$ C.R. $=3.0 ;(b)$ influence of the C.R.'s: $1-$ C.R. $=3.0 ; 2-2.5 ; 3-2.0 ; 4-1.5$; and $5-$ C.R. $=1$

for the 'military patrol, bomber, and transport' category from AAA (dashed line). As there is only a very small difference between the two curves and as it is conservative for the range of takeoff weights to be expected, the coefficients of AAA are adopted here. It is thus assumed that the twin fuselage layout has no considerable influence on the aircraft empty weight.

In a next step, the influence of the use of liquid hydrogen is assessed and new regression coefficients are determined based on available data [7, 12]. The result is shown in Fig. $2 b$. The solid line in the figure represents the hydrogenfuelled aircraft while the dashed line shows the correlation for military patrol, bomber, and transport aircraft from AAA. The figure clearly shows that the use of hydrogen leads to a significant increase in aircraft empty weight. This increase mainly arises from the thick insulaton needed to store the cryogenic fuel.

In a final step, an additional load alleviation system is incorporated in the design to reduce the takeoff weight to a (relatively) reasonable value. Such a system would namely allow one to certify the mothership to $2 g$ 's instead of the typical $2.5 \mathrm{~g}$ used for civil airliners. This will obviously lead to a reduction of the aircraft structural weight and thus also of the empty weight. The regression coefficient $A$ is therefore slightly reduced $[4,13]$ which leads to a final value of 0.2924 for $A$ and 0.9907 for $B$.

Based on the mission specification of Table 1 and the regression coefficients $A$ and $B$, the different weights of the aircraft can be determined as shown in Fig. $3 a$. For a collection ratio of 3.0, the TOGW of $969.4 \mathrm{t}$ is obtained for an empty weight of $563.7 \mathrm{t}$. Even though this TOGW seems very high, it is very much comparable to the 900-ton TOGW of the Herakles concept [14]. As the aircraft collects oxygen during cruise, the maximum flight weight is attained just before the launch of the orbiter, as shown in Fig. 3. The calculated maximum weight is $1035.8 \mathrm{t}$. In total, $213.8 \mathrm{t}$ of hydrogen are needed for the mission. 


\subsection{Performance Sizing}

Now, when the TOGW of the aircraft is known, different performance sizing requirements can be calculated, which leads to the selection of the wing area and takeoff thrust. To be able to do that, the drag polars need to be estimated first. Obviously, this is an iterative process and the values mentioned here are the final results of this iteration. First, the drag polar estimation for the mothership and the orbiter are shortly discussed. Then, the performance sizing of the mothership is tackled and the final configuration is selected.

\subsubsection{Drag Polar Estimation}

In order to estimate the drag polar of the aircraft in the different configurations (clean, with orbiter, takeoff flaps, etc.), the wing area and position needs to be fixed first. Several requirements immediately limit the options for the wing choice. First of all, the mothership must have a high wing since the orbiter will be attached underneath the central part of the wing (see Fig. 4b). This also fixes the distance between the two fuselages as a sufficient spacing between the fuselages and the orbiter is needed to avoid excessive interference drag. As a spanwise separation of the fuselages should ideally be around $35 \%$ of the wing span to minimize wing weight [9], the span thus also is fixed. Finally, the performance sizing requirements also impose limits upon the wing loading and wing area of the aircraft. These limits, together with the TOGW determined previously, thus fix the gross wing area of the mothership which is set at $1414 \mathrm{~m}^{2}$. As the wing span was set at $114 \mathrm{~m}$, the wing has an aspect ratio of 9.20.

The wing size and shape are known, so the drag polar of the clean aircraft (no orbiter, no flaps deployed, landing gear retracted) can be determined. A parabolic drag polar is assumed, following the methodology imposed by AAA:

$$
C_{D}=C_{D_{0}}+\Delta C_{D_{0}}+\frac{C_{L}^{2}}{\pi A R e}
$$

where the zero-lift drag coefficient $C_{D_{0}}$ is calculated using the so-called parasite drag area [5], the drag increment due to compressibility $\Delta C_{D_{0}}$ is taken as 10 drag counts, while the Oswald efficiency factor is set to 0.83 . A small reduction compared to the normal values is taken to reflect the effect of the two fuselages on the wing spanwise lift distribution. The drag polar equation thus becomes

$$
C_{D}=0.0112+0.0413 C_{L}^{2}
$$

which leads to a maximum lift-to-drag ratio of approximately 23 at a lift coefficient around 0.5 .

Of course, the presence of the orbiter will change the drag polar of the aircraft, as it will add skin friction as well as interference drag. The additional skin friction 


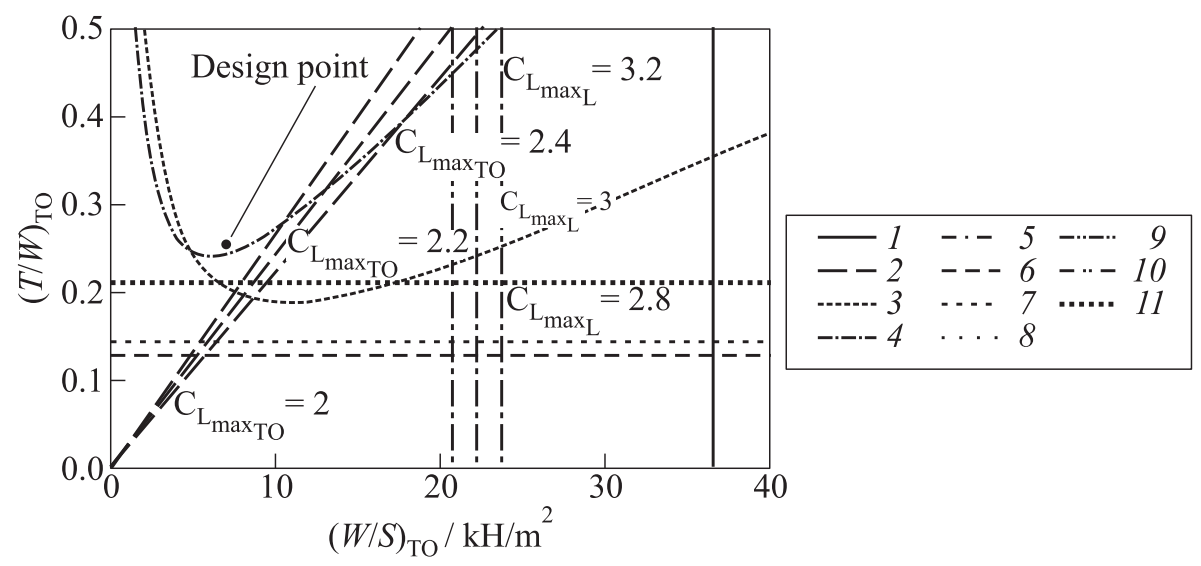

(a)

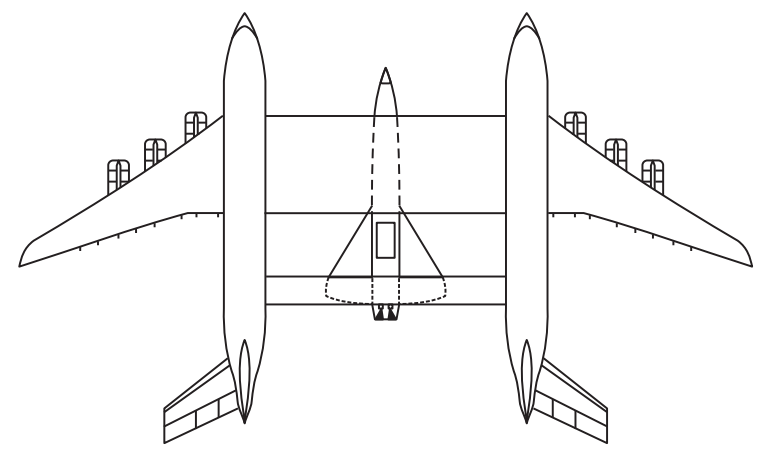

(b)

Figure 4 The matching plot and the final configuration of the mothership: $(a)$ the matching plot $\left(1-\right.$ stall speed; 2 - takeoff distance, $\Delta T=0{ }^{\circ} \mathrm{C} ; 3$ - maximum cruise speed; 4 - sustained $\mathrm{g} /$ turn rate; 5 - landing distance, $\Delta T=0{ }^{\circ} \mathrm{C} ; 6-$ climb OEI (one engine inoperative); 7 - climb OEI transition; 8 - climb OEI second segment; 9 - climb OEI enroute; 10 - climb OEI approach; and 11 - climb AEO (all engines operating) landing) and $(b)$ the final configuration

drag was calculated for the orbiter and converted back to the wing gross area, which lead to a drag increase of around 150 drag counts [4, 13]. This resulted in the following drag polar for the mothership and orbiter, without flaps and landing gear deployed:

$$
C_{D}=0.0262+0.0418 C_{L}^{2} .
$$




\subsubsection{Performance Sizing and Selection of Final Configuration}

In order to select the appropriate wing loading and thrust-to-weight ratio for the envisaged mission, several performance requirements are imposed on the design. All performance requirements are then graphically represented in the so-called matching plot. Below, the different imposed requirements are concisely covered and the final matching plot is given.

Even though for the jet transport aircraft, normally no stall speed requirements are imposed, they were, nevertheless, evaluated here to assure that the launching system with all LOX collected can land in case of an emergency without encountering stall problems. The stall speed was therefore evaluated at the maximum weight of the aircraft at the height of the Vandenberg air force base. As the aircraft obviously needs to be able to takeoff from its base, the field length of the Vandenberg air force base was taken for the takeoff distance sizing requirement too. A similar approach was taken for the landing distance. Again, as for the stall speed sizing, the maximum weight of the launcher was used for this requirement. Furthermore, the climb gradients imposed by the FAR25 regulations were also applied to this design to ensure a sufficient climb gradient with one engine failed. Finally, to add a small thrust margin to the design, a manoeuvering requirement for a sustained turn at a bank angle of $48^{\circ}$ was imposed (load factor $n$ of 1.5) to allow the necessary maneuvers to launch the orbiter. As this required a very high thrust rate, however, a special engine setting was considered, adding $17 \%$ of thrust for a short period of time (just before the launch of the second stage). All these requirements lead to the matching plot shown in Fig. $4 a$.

From the figure, it is clear that the thrust is set by the maneuvering requirement, despite the high engine rating taken into account. All other requirements will normally not pose problems. The final design point for the first-stage subsonic aircraft is thus

$$
\left(\frac{W}{S}\right)_{\mathrm{TO}}=685.72 \frac{\mathrm{kg}}{\mathrm{m}^{2}} \quad \text { and } \quad\left(\frac{T}{W}\right)_{\mathrm{TO}}=0.2425
$$

which leads to a required thrust per engine at takeoff of $384.07 \mathrm{kN}$. As the GE9090B engine delivers, according to calculations with Gasturb [15], an installed thrust of almost $395 \mathrm{kN}$ a thrust surplus of $3 \%$ is thus attained.

In a final step, longitudinal static stability calculations were executed to determine the relative position of the different aircraft components. Obviously, the weight of the different components needs to be determined before this can be attempted. In a conceptual design, this is often done based upon average component weights [6]. In consultation with Dr. Roskam, the component weight fractions of the Boeing 747-100, the C-5A, the C-133 A, and the Brittania 300 are used as the base for this calculations. The fractions are adapted to reflect the twin fuselage configuration and the use of hydrogen. With the resulting 
component weights, a check is made for the c.g. (center of gravity) excursion and the wing longitudinal position on the fuselage is changed until a suitable (small) excursion is obtained. An excursion of only $2 \%$ of the wing mean aerodynamic chord was obtained which gives sufficient margin seen the uncertanties on the computed weight fractions. The resulting configuration is shown on Fig. $4 b$. As can be seen from the figure, the horizontal tail was only located on the outside of the fuselages. This is done to ensure that the control surfaces are not in the wake of the orbiter. The fuselages shown are based on the B777-300.

\subsection{Influence of the Collection Ratio on the Mothership}

Since all previous data was obtained using a collection ratio of 3.0, the influence of this collection ratio, the amount of LOX collected per kilogram of hydrogen fuel used, on the masses and performance of the first stage aircraft is assessed in a final step of the conceptual design of the mothership. In this assessment, only the collection ratio is changed, all other mission and performance parameters are held constant as far as possible. For the lower collection ratios, the cruise time before the launch of the second stage, however, had to be increased to collect all $210 \mathrm{t}$ of LOX. The resulting weights for the different collection ratios are shown in Fig. $3 b$.

As shown in the figure, the cruise length was increased for collection ratios of 1.5 and 1.0. The resulting increase in cruise length has a significant influence on the maximum mass of the mothership, as is shown in the figure. For collection ratios between 2 and 3 , the ratio, however, has only a small influence on the TOGW. A collection ratio of 2.0 leads to an increase of only approximately $2 \%$ of the TOGW which is obviously acceptable. As the takeoff weight is changed, the performance sizing should be checked for the different collection ratios. The results for the design point are summarized in Table 2 . The table shows that the GE90-90B engine can be used for collection ratios between 2.0 and 3.0. If

Table 2 Design point for the different collection ratios

\begin{tabular}{ccc}
\hline C.R. & $\left(\frac{W}{S}\right)_{\mathrm{TO}}, \mathrm{kg} / \mathrm{m}^{2}$ & $T_{\mathrm{TO}} /$ engine, $\mathrm{kN}$ \\
\hline 3.0 & 685.72 & 384.07 \\
2.5 & 691.05 & 386.42 \\
2.0 & 696.29 & 389.35 \\
1.5 & 749.02 & 415.37 \\
1.0 & 917.22 & 521.36 \\
\hline
\end{tabular}


the ratio is lowered below 2.0, a more powerful engine is, however, needed. For a collection ratio as low as 1 , a new wing should also be chosen, as the obtained wing loading exceeds the current limits.

\section{THE LOX COLLECTION AND AIR SEPARATION PLANT}

The separation plant cycle takes atmospheric air, deeply cools it, and separates it into nitrogen and oxygen to the best practical extent. The plant is located on the mothership, where it causes a minimal mass penalty and could recuperate evaporated hydrogen from the first stage. Even though a reasonable insulation can limit boil-off losses down to very low values, recuperating the evaporated hydrogen and using it for propulsion of the mothership will improve the overall efficiency of the two-stage launcher. On the one hand, the separation plant is coupled to the fuel lines of the propulsion system of the mothership to exploit the cooling capacity of the $\mathrm{LH}_{2}$, which also leads to a small improvement of the specific fuel consumption of the first stage engines. The LEA produced by the collection plant is, on the other hand, stored on the second stage for later use as oxidizer for the LH2-LOX rocket engines. For reasons linked to separation system physics, the purity of the LEA is limited to the $90 \%-95 \%$ range and the total amount of oxygen collected amounts to about $70 \%$ of the oxygen present in the air ingested by the separation plant.

Below, first the choices taken for the plant design are elucidated. Then, the separation unit itself as well as the heat exchangers are described. Finally, the arrangement of the different units in the plant cycle is covered and the plant performance is assessed.

\subsection{Air Separation Plant Design Choices}

In the plant design, a modular practical solution was envisaged compared to optimized systems. Even though the latter could be slightly more efficient, light and compact, they would namely give much less flexibility for practical development [16]. Whenever possible, readily available, existing elements were used. Following this design line was possible for most of the elements, including the heat exchangers, leading to reasonable masses and volumes. For the separation unit itself, this was, however, not possible. Nonetheless, the separator has been split in three almost identical base units with a volume of approximately $1 \mathrm{~m}^{3}$. These units are combined in a modular manner to build up the complete separator, which could therefore be assembled to obtain different layouts [17]. 


\subsection{Air Separation Plant}

The operating principle behind the separation process is distillation. A classical distillation process would, however, be too bulky and heavy to be stored in a flying structure. A nonclassical separation device has therefore been developed: a centrifugally enhanced distillation unit. Historically, the first designs and even prototypes of such a device date back from the 1950s and 1960s [18]. Further studies were performed in the United States in the 1990s, but with only limited experimental work [19]. Other studies were also executed in Russia and Europe on a vortex tube device [20]. More recently, there is a renewal of interest in the concept in the U.S. where the company Andrews has received funding under a DARPA contract for long term research on the topic [21], even up to experimental work. Unfortunately, only a very limited amount of information is diffused [22].

The centrifugally enhanced distillation test unit design developped by the authors' team uses high porosity materials to obtain sufficient contact surface between liquid and gaseous air in order to distillate the media sufficiently fast with a compact and light device. Using this type of fine structures is only possible under high acceleration forces $(500 g-2000 g)$, provided by the rotation of the device. The unit that has been designed and built by the team has already shown some promising results in the first tests. Furthermore, the study provided better phenomenological understanding of the encountered phenomena that allowed the prediction of the performance of a practical unit $[17,23]$.

\subsection{Heat Exchangers}

While the separator proves to be the most prospective element, the heat exchangers are the most critical components for the safety, the mass, and the performance of the complete cycle. They have to provide a high heat transfer capability with limited pressure drop for a limited mass and volume. Suitable commercially available heat exchangers were identified under the AHEX programme, funded by the ESA GSTP programme, and in collaboration with IberEspacio and Techspace Aero. As far as possible, aluminum has been considered, since it allows to get a significant mass reduction for a robust design. As the maximum operating temperature of the heat exchangers is limited, the usage of aluminum entails no restrictions. The material is furthermore compatible with the use of $\mathrm{H}_{2}$ as most aluminum alloys do not suffer from hydrogen embrittlement problems. Additionally, they show good tightness specifications for the envisaged designs, which could otherwhise prove to be a major safety concern.

\subsection{Air Separation Plant Arrangement}

Figure 5 shows the arrangement of the different components for the simple 'proof of concept' cycle. Despite not being optimized, the cycle has a sufficient level 


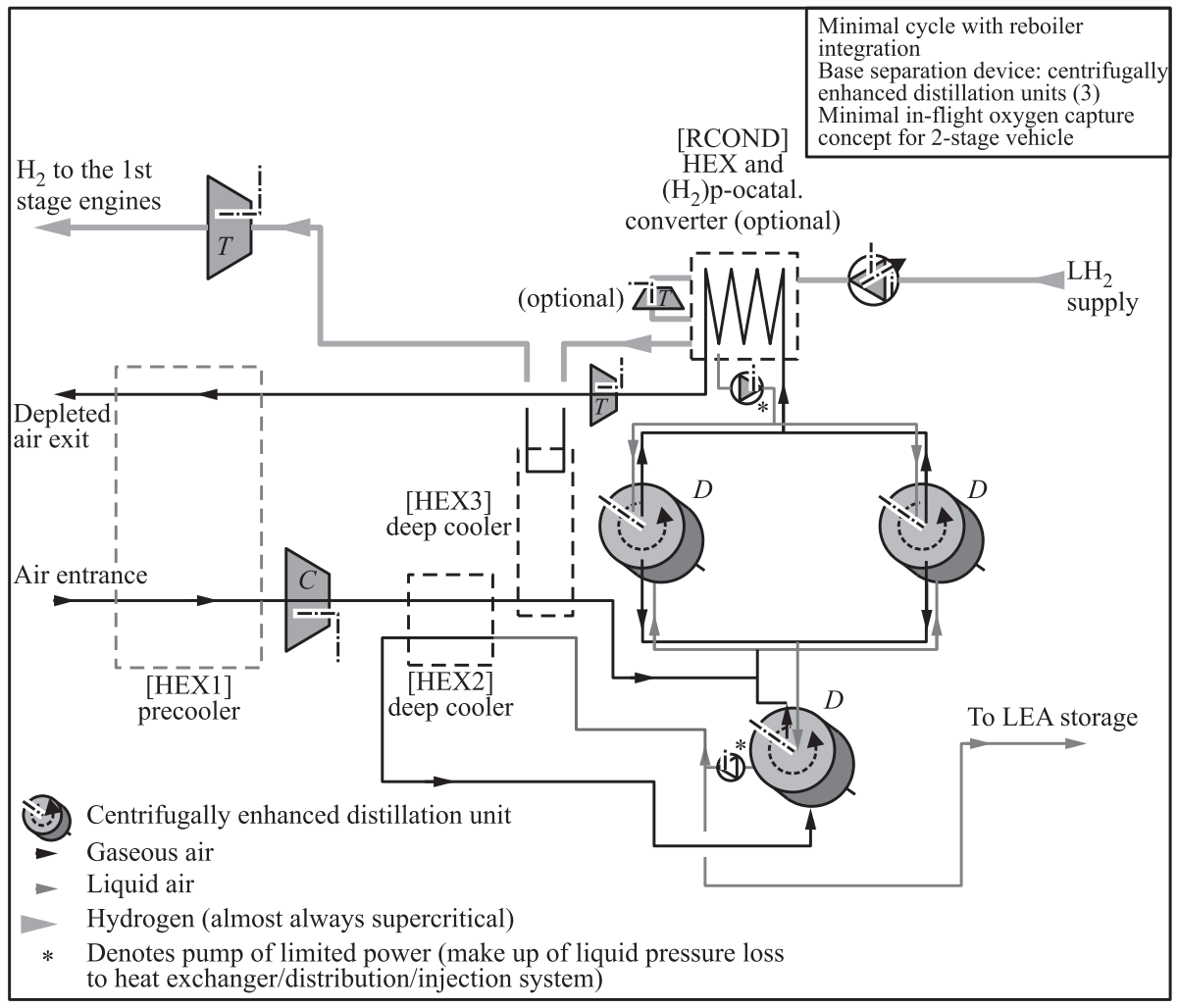

Figure 5 Separator plant arrangement

of performance and relies on available and modular technological solutions. The air is cooled in a sequence of heat exchangers, operating close to saturation. As the third exchanger leads to a slightly superheated gas at the entrance of the actual distillation system, it could possibly be eliminated. However, a more accurate and complete check of the performance degradation this entails, should be made first. The three distillation units are interconnected by small liquid pumps to make up for pressure losses of the different components and, mainly, to balance the pressure differences between the units. To allow the gas to flow to the top part (rectification), the lower part of the distillation plant (stripping) is kept in slight relative overpressure. At the moment, the operating pressure is set at 3 bar, which is far from optimal and tends to lead to relatively bulky and heavy units. It does, however, greatly simplify the construction of the first prototype while huge improvement potentials remain present. 


\subsection{Collection Plant Performance and Mass}

The performance of the plant is described in terms of C.R. (mass of oxygen collected per mass of hydrogen consumed), LEA purity, oxygen recovery factor, mass, and volume. With the current design, a C.R. of 2.4 seems easily attainable. If the para-ortho conversion is exploited and some form of $\mathrm{H}_{2}$ expansion is used to increase the hydrogen cooling capacity, a higher value could even be achieved. For a plant that produces around $10 \mathrm{~kg} / \mathrm{s}$ of LEA, a mass of approximately $10 \mathrm{t}$ is estimated, based on existing heat exhanger data and performance analysis [24]. The precooling and deep cooling devices are the major contributors to the plant in terms of volume and mass. For a noncompact arrangement, 30 to $40 \mathrm{~m}^{3}$ is expected to be required. Even though the plant is at the moment not optimized, both values are clearly acceptable, showing that the concept for the first stage is not standing on an edge. Its main practical difficulty would be the rather large size and the associated development costs.

\section{DESCRIPTION OF THE AIR SEPARATION TEST UNIT}

Among the different options, a separation based on controlled distillation was chosen and used as a baseline for both system studies and experimental testing. For the process to be fast enough, mass transfer at the liquid/gas interface has to be fast. It is therefore necessary to increase the contact surface between liquid and gas in the device. In a classical tray column, this is done by finely bubbling the gas in the liquid through the tray plate, but for different reasons, that solution did not appear attractive when attempting to make it compact. Alternative to tray use in columns is the use of a porous material - a so-called packing — where liquid flow trickles against gas flow. To increase the contact surface, the characteristic dimension of the porous material - the packing - has to be reduced dramatically from a usual value (at least by a factor of about 10), which causes drowning of the column during its operation, due to capillarity forces in the packing. The solution is to use a rotating device, allowing to reach accelerations above some hundred $g$ up to several thousand $g$. Much finer packings can be used and the device can be made compact and weight efficient. Although less efficient for a given set of geometric parameters, a random packing has been chosen as a separation bed [25]: metal foam (Fig. 6). It offers the best combination of properties and should result in the lightest and most compact distillation system. A usual culprit, when distillating air, is the presence of argon that can be difficult to separate from oxygen. Argon concentration in air and the limited plant ability to separate oxygen and argon combine to fulfil the specification for oxygen purity delivered (90\% in mass) without great difficulty. 


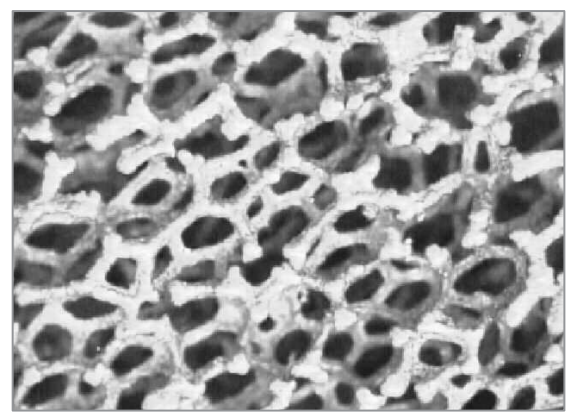

Figure 6 Internal structure of the metal foam used as separator bed. The specific surfaces commercially available range from 500 to $5000 \mathrm{~m}^{2} / \mathrm{m}^{3}$. This material has also high porosity while preserving significant mechanical properties. The material shown in the picture has a specific surface of about $2500 \mathrm{~m}^{2} / \mathrm{m}^{3}$ and the diameter of the structural "strut" is about $0.1 \mathrm{~mm}$

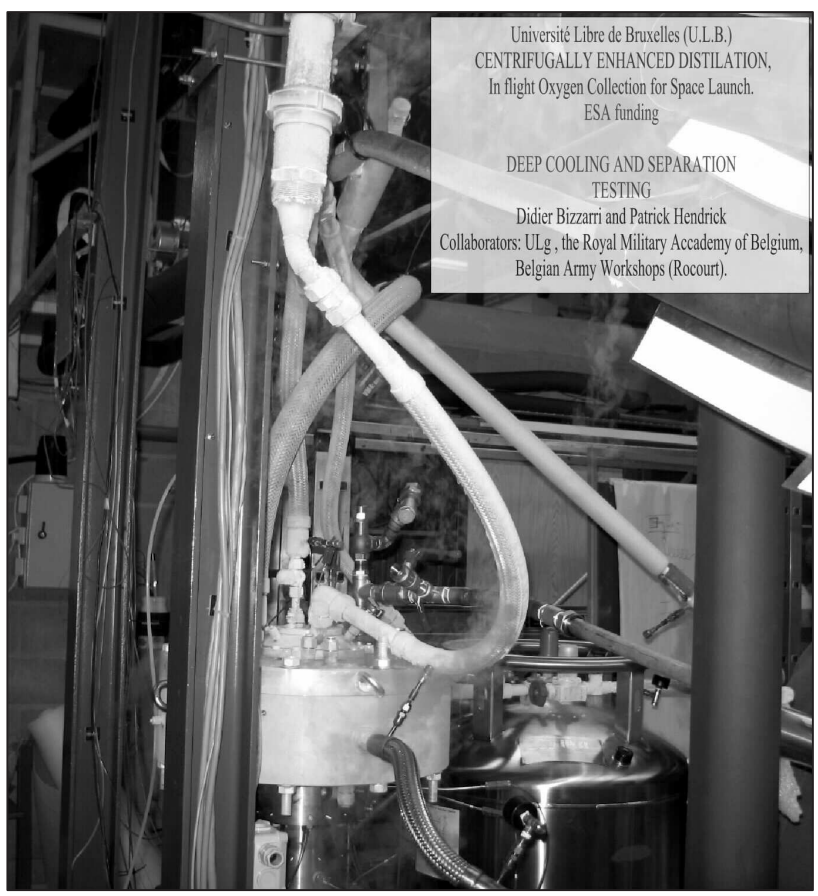

Figure 7 Test rig during separation testing 
A subscale test setup has been designed and built to prove the concept based on those basic ideas [26]. First tests with the unit have shown separation by delivering oxygen concentrated liquid air. Figure 7 shows the unit during operation. Performance quantification is under way.

On the theoretical side, estimation of performance (purity and throughput) based on existing data and theoretical approach have been performed along and allow not only to understand the phenomenology and to give first guesses on results, but also provide the tools to scale results to smaller or bigger units.

\section{VEHICLE - SEPARATION PLANT INTEGRATION}

Three different vehicle-separation plant integration schemes could be envisaged as shown in Fig. 8. In a first scheme, the plant would be housed in the wing root, in between the two fuselages. Even though, in theory, the central wing would be spacious enough to accept a separation plant, the current system would have to be redesigned in smaller elements. This type of installation would furthermore not be practical for mounting inspection and maintenance. A second option is to split the separation plant in two halves, which also requires the design of smaller units. Each half would then be placed in the nose of both fuselages, which would furthermore require an additional extension of one of the fuselages for the cockpit. A third, probably more practical option, would be the addition of a third fuselage, mounted on top of the central wing. This third fuselage would

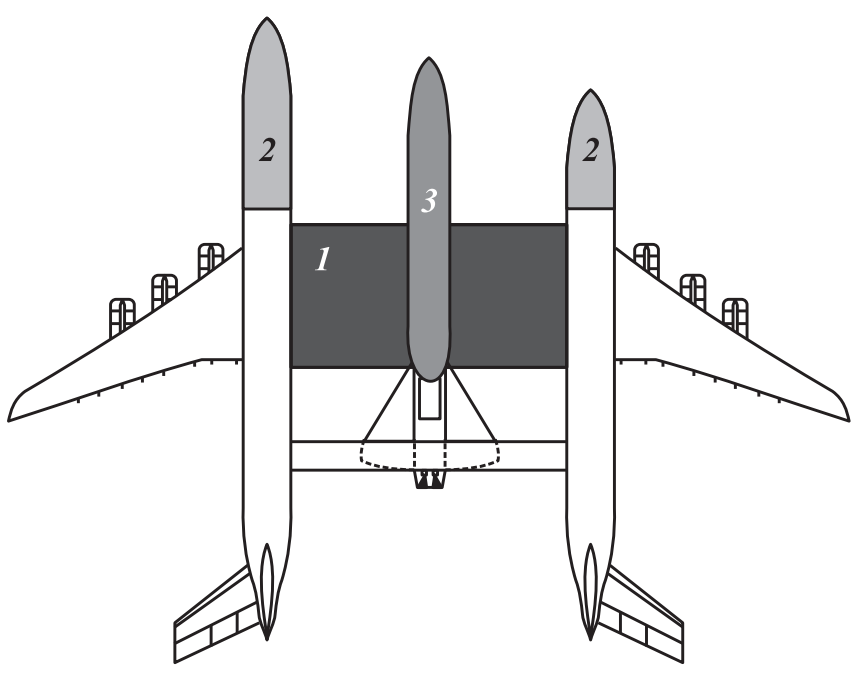

Figure 8 Different vehicle-separation plant integration schemes 
accommodate both the separation plant and the cockpit. As the collection plant would be very close to the orbiter, this configuration would furthermore limit LEA evaporating losses while feeding the orbiter with the oxidizer. A slightly better insulation for the $\mathrm{LH}_{2}$ lines could, however, be required. The proximity of the cockpit and the separation plant would additionally allow an easy access to the plant in case of operational problems. With inhabited orbiters, the orbiter staff could even stay in the mothership until just before the launch.

Even though all three configurations seem to be feasible, the latter one is prefered for prototype validation. Nevertheless, integration of the plant into the mothership does not pose significant problems.

\section{CONCLUDING REMARKS}

A twin-fuselage aircraft has been designed as the first stage of a two-stage-toorbit space launcher. This mothership collects oxygen for the orbiter during its $6,250 \mathrm{~km}$ cruise phase to the launch point. A preliminary estimation of the mass and drag polar has shown that the concept would be feasible for collection ratios between two and three, which is quite low.

The collection plant has been designed and sized and is shown to be integrable in the system provided some modifications are made. The integration of a prototype air separation, liquefaction plant for oxygen collection has shown to be possible while a strong potential for separator plant mass and volume reduction, as well as performance increase, would still be feasible.

\section{REFERENCES}

1. Hendrick, P., and A. Mignon. 2000. Utilization of existing large subsonic aircraft elements to implement in-flight LOX collection. AIAA Paper No. 2000-3107.

2. DARCorporation, Advanced Aircraft Analysis Version 2.3 help files. 2001. DARCorporation.

3. DARCorporation. http://www.darcorp.com.

4. Verstraete, D., and P. Hendrick. 2003. Preliminary design of a multipurpose twin fuselage aircraft. National Congress on Theoretical and Applied Mechanics. Ghent, Belgium.

5. Roskam, J. 1997. Airplane design. Part I: Preliminary sizing of airplanes. DARCorporation.

6. Roskam, J. 1997. Airplane design. Part II: Preliminary configuration design and integration of the propulsion system. DARCorporation.

7. Brewer, G. D. 1991. Hydrogen aircraft technology. CRC Press.

8. Hendrick, P. 2002. Preliminary design of reusable space planes using in-flight oxygen collection. Ph.D. Thesis. RWTH Aachen. 
9. Houbolt, J. 1982. Why twin-fuselage aircraft? J. Astronautics Aeronautics 20(4):26-35.

10. Moore, J. W. 1982. Multibody aircraft study. Vol. 1. NASA-CR-165829-Vol-1.

11. Chiesa, S., M. Di Siuva, and P. Maggiore. 2000. The double-fuselage layout: A preliminary case study of a possible way of reducing the development costs for new high capacity aircraft? Proc. Instn. Mech. Engrs. 214(Pt. G):85-95.

12. Brewer, G.D., R.E. Morris, R.H. Lange, and J.W. Moore. 1975. Study of the application of hydrogen fuel to long-range subsonic transport aircraft. NASACR132559 .

13. Verstraete, D., and P. Hendrick. 2003. Preliminary design of an ACES TSTO air launch vehicle. AIAA Paper No. 2003-6997.

14. The Herakles launch vehicle. http://www.astronautix.com/lvfam/winged.htm

15. Gasturb 9. http://www.gasturb.de.

16. Bizzarri, D., and P. Hendrick. 2006. Analysis of minimal in-flight oxygen collection cycle for 2 stage launchers. AIAA Paper No. 2006-8097.

17. Bizzarri, D., G. Heyen, P. Hendrick, and P. Ngendakumana. 2005. Integration of vehicle, propulsion system and separation unit designs for a launcher using in-flight oxygen collection. AIAA Paper No. 2005-3421.

18. Leingang, J. L., L. Q. Maurice, and L. Carreiro. 1996. Wright laboratory, in-flight oxidiser collection systems for airbreathing space boosters, Developments in HighSpeed-Vehicle Propulsion Systems. AIAA book. 165(Ch. 7):333-384.

19. Acharya, A., C.F. Gottzmann, and J. J. Nowobilski. 1990. Airborne rotary air separator study. Union Carbide Industrial Gases Inc. NASA CR-189099.

20. Hendrick, P., F. Breugelmans, B. Marquet, M. Saint-Mard, M. Strengnart, P. Ngendakumana, and D. Bizzarri. 2000. Fully and partially reusable TSTO launchers using in-flight LOX collection. Morioka, Japan. ISTS 2000-g-09.

21. Andrews, J.E., and D.G. Andrews. 2001. Designing reusable launch vehicles for future space markets. 52nd Astronautical Congress (International) Proceedings. Toulouse, France. IAA-01-IAA.1.1.07.

22. Andrews Advanced technologies, Air Collection and Enrichment System. http://www.andrews-space.com/.

23. Heyen, G. 2003. Oxygen separation for advanced rocket engine Rotary Distillation Separator for in-flight LOX collection. Simulation study. Report 2. Liège University, LASSC.

24. Dardenne, L., and P. Ngendakumana. 2006. Study of precooler for in-flight oxygen collection. ESA Report, WorkPackage 7000. Technical Note TN6 (Issue 1): Second Breadboard Design. Université de Liège \& Université Libre de Bruxelles.

25. Bizzarri, D., and P. Hendrick. 2003. Study and development of a air distillation subscale unit for in-flight oxygen collection. AIAA Paper No. 2003-6930.

26. Bizzarri, D., and P. Hendrick. 2005. Experimental study of an in-flight air separation device. AIAA Paper No. 2005-3422. 